\title{
Approximate Solution of Volterra-Stieltjes Linear Integral Equations of the Second Kind with the Generalized Trapezoid Rule
}

\author{
Avyt Asanov, ${ }^{1}$ Elman Hazar, ${ }^{2,3}$ Mustafa Eroz, ${ }^{2}$ Kalyskan Matanova, ${ }^{1}$ and Elmira Abdyldaeva ${ }^{1}$ \\ ${ }^{1}$ Department of Mathematics, Kyrgyz-Turkish Manas University, Bishkek, Kyrgyzstan \\ ${ }^{2}$ Department of Mathematics, Sakarya University, Sakarya, Turkey \\ ${ }^{3}$ Department of Applied Mathematics and Informatics, Kyrgyz-Turkish Manas University, Bishkek, Kyrgyzstan
}

Correspondence should be addressed to Elman Hazar; ealiyev@sakarya.edu.tr

Received 21 June 2016; Revised 15 August 2016; Accepted 24 August 2016

Academic Editor: Soheil Salahshour

Copyright (C) 2016 Avit Asanov et al. This is an open access article distributed under the Creative Commons Attribution License, which permits unrestricted use, distribution, and reproduction in any medium, provided the original work is properly cited.

\begin{abstract}
The numerical solution of linear Volterra-Stieltjes integral equations of the second kind by using the generalized trapezoid rule is established and investigated. Also, the conditions on estimation of the error are determined and proved. A selected example is solved employing the proposed method.
\end{abstract}

\section{Introduction}

Various issues concerning Volterra and Volterra-Stieltjes integral equations were studied in [1-13]. Some practical and theoretical investigations were made in paper [1] for nonclassical Volterra integral equations of the first kind. Also, the approximate solution for the integral equation considered is obtained. In paper [2], various inverse problems including Volterra operator equations were studied. Some properties for Volterra-Stieltjes integral operators were given in [3]. In the studies [6,7], existence and uniqueness of the solutions were given for Volterra integral and Volterra operator equations of the first and the second kinds. In papers $[4,6]$, quadratic integral equations of Urysohn-Stieltjes type and their applications were investigated. Various numerical solution methods for integral equations were presented in the studies [8-13]. The notion of derivative of a function by means of a strictly increasing function was given by Asanov in [14]. In the study [15], the generalized trapezoid rule was proposed to evaluate the Stieltjes integral approximately by employing the notion of derivative of a function by means of a strictly increasing function.

In this study, we investigate the numerical solution of linear Volterra-Stieltjes integral equations of the second kind by using the generalized trapezoid rule. Therefore, we need the concept of the derivative defined in the works $[14,15]$ and theorems connected with it.

\section{Approximating Volterra-Stieltjes Integral Equations}

Consider the linear integral equation of the second kind

$$
u(x)=\int_{a}^{x} K(x, s) u(s) d \varphi(s)+f(x), \quad x \in[a, b],
$$

where $K(x, s)$ is a given continuous function on $G=\{(x, s)$ : $a \leq s \leq x \leq b\}, f(x)$ are given continuous functions on $[a, b], \varphi(s)$ is a given strictly increasing continuous function on $[a, b]$, and $u(x)$ is the sought function on $[a, b]$.

Definition 1. The derivative of a function $f(x)$ with respect to $\varphi(x)$ is the function $f_{\varphi}^{\prime}(x)$, whose value at $x \in(a, b)$ is the number

$$
f_{\varphi}^{\prime}(x)=\lim _{\Delta \rightarrow 0} \frac{f(x+\Delta)-f(x)}{\varphi(x+\Delta)-\varphi(x)},
$$


where $\varphi(x)$ is a given strictly increasing continuous function in $(a, b)$.

If the limit in (2) exists, we say that $f(x)$ has a derivative (is differentiable) with respect to $\varphi(x)$. The first derivative $f_{\varphi}^{\prime}(x)$ may also be a differentiable function with respect to $\varphi(x)$ at every point $x \in(a, b)$. Then, its derivative

$$
f_{\varphi}^{\prime \prime}(x)=\left(f_{\varphi}^{\prime}(x)\right)_{\varphi}^{\prime}
$$

is called the second derivative of $f(x)$ with respect to $\varphi(x)$. Consequently, the $n$th derivative of $f(x)$ with respect to $\varphi(x)$ is defined by

$$
f_{\varphi}^{(n)}(x)=\left(f_{\varphi}^{(n-1)}(x)\right)_{\varphi}^{\prime}
$$

We need the following theorem which is given in [15].

Theorem 2. Let $\varphi(x)$ and $\psi(x)$ be two strictly increasing continuous functions on $[a, b]$ and $f_{\varphi}^{\prime \prime}(x), f_{\psi}^{\prime \prime}(x) \in C[a, b]$. Then,

$$
\begin{aligned}
\left|I-A_{n}\right|= & \frac{M_{0}}{12}(\varphi(b)-\varphi(a))\left(\omega_{\varphi}(h)\right)^{2} \\
& +\frac{M_{0}^{\prime}}{12}(\psi(b)-\psi(a))\left(\omega_{\psi}(h)\right)^{2},
\end{aligned}
$$

where

$$
\begin{aligned}
& I=\int_{a}^{b} f(x) d \varphi(x)-\int_{a}^{b} f(x) d \psi(x), \\
& M_{0}=\left\|f_{\varphi}^{\prime \prime}(x)\right\|_{C}=\sup _{x \in[a, b]}\left|f_{\varphi}^{\prime \prime}(x)\right|, \\
& M_{0}^{\prime}=\left\|f_{\psi}^{\prime \prime}(x)\right\|_{C}=\sup _{x \in[a, b]}\left|f_{\psi}^{\prime \prime}(x)\right| \\
& \omega_{\varphi}(h)=\sup _{|x-y| \leq h}|\varphi(x)-\varphi(y)|, \\
& \omega_{\psi}(h)=\sup _{|x-y| \leq h}|\psi(x)-\psi(y)|, \\
& A_{n} \quad \\
& =\frac{1}{2} \sum_{i=1}^{n}\left[f\left(x_{i}\right)+f\left(x_{i-1}\right)\right]\left[\varphi\left(x_{i}\right)-\varphi\left(x_{i-1}\right)\right] \\
& \quad-\frac{1}{2} \sum_{i=1}^{n}\left[f\left(x_{i}\right)+f\left(x_{i-1}\right)\right]\left[\psi\left(x_{i}\right)-\psi\left(x_{i-1}\right)\right]
\end{aligned}
$$

and $x_{i}=a+i h, i=0,1, \ldots, n, h=(b-a) / n, n \in N(N$ denotes the set of natural numbers).

Corollary 3. Let $\varphi(x)$ be a strictly increasing continuous function on $[a, b], \psi(x)=0$ for all $x \in[a, b]$ and $f_{\varphi}^{\prime \prime}(x) \epsilon$ $C[a, b]$. Then,

$$
\begin{aligned}
\left|I-A_{n}\right| & \leq \frac{M_{0}}{12}(\varphi(b)-\varphi(a))\left(\omega_{\varphi}(h)\right)^{2}, \\
\left|I_{i}-M_{i}\right| & \leq \frac{M_{0}}{12}\left(\varphi\left(x_{i}\right)-\varphi\left(x_{i-1}\right)\right)^{3}, \quad i=1,2, \ldots, n,
\end{aligned}
$$

where

$$
\begin{aligned}
I_{i} & =\int_{x_{i-1}}^{x_{i}} f(x) d \varphi(x), \\
M_{i} & =\frac{1}{2}\left[f\left(x_{i}\right)+f\left(x_{i-1}\right)\right]\left[\varphi\left(x_{i}\right)-\varphi\left(x_{i-1}\right)\right] .
\end{aligned}
$$

Theorem 4. Let $\varphi(x)$ be a strictly increasing continuous function on $[a, b], K(x, s) \in C(G)$, and $f(x) \in C[a, b]$. Then, the integral equation (1) has a unique solution $u(x) \in C[a, b]$ and

$$
\|u(x)\|_{C} \leq c_{1}\|f(x)\|_{C},
$$

where $c_{1}=\exp \left\{K_{0}(\varphi(b)-\varphi(a))\right\}$ and $K_{0}=\|K(x, s)\|_{C}=$ $\sup _{(x, s) \in G}|K(x, s)|$.

Then, we will need the following theorem which is given in [16].

Theorem 5. Let $F(x, s), F_{\varphi(x)}^{\prime}(x, s) \in C(G), \varphi(x)$ be strictly increasing continuous functions on $[a, b]$, and $P(x)=$ $\int_{a}^{x} F(x, s) d \varphi(s), x \in[a, b]$. Then,

$$
P_{\varphi(x)}^{\prime}(x)=F(x, x)+\int_{a}^{x} F_{\varphi(x)}^{\prime}(x, s) d \varphi(s),
$$

$$
x \in[a, b] \text {, }
$$

where

$$
\begin{aligned}
F_{\varphi(x)}^{\prime}(x, s)= & \lim _{\Delta x \rightarrow 0} \frac{F(x+\Delta x, s)-F(x, s)}{\varphi(x+\Delta x)-\varphi(x)}, \\
(x, s) \in\{(x, s): a<s<x<b\}, & \\
P_{\varphi(x)}^{\prime}(a)= & \lim _{\Delta x \rightarrow 0+} \frac{P(a+\Delta x)-P(a)}{\varphi(a+\Delta x)-\varphi(a)}, \\
P_{\varphi(x)}^{\prime}(b)= & \lim _{\Delta x \rightarrow 0-} \frac{P(b+\Delta x)-P(b)}{\varphi(b+\Delta x)-\varphi(b)} .
\end{aligned}
$$

Corollary 6. Let $u(x) \in C[a, b]$ be a solution of the integral equation (1), $K_{\varphi(x)}^{\prime}(x, s) \in C(G)$, and $f_{\varphi(x)}^{\prime}(x) \in C[a, b]$. Then, $u_{\varphi(x)}^{\prime}(x) \in C[a, b]$ and

$$
\begin{aligned}
u_{\varphi(x)}^{\prime}(x)= & K(x, x) u(x)+\int_{a}^{x} K_{\varphi(x)}^{\prime}(x, s) u(s) d \varphi(s) \\
& +f_{\varphi(x)}^{\prime}(x),
\end{aligned}
$$

where $x \in[a, b]$.

Corollary 7. Let $u(x) \in C[a, b]$ be a solution of the integral equation (1), $K_{\varphi(x)}^{\prime \prime}(x, s) \in C(G), K_{\varphi(x)}^{\prime}(x, x) \in C[a, b]$, and $f_{\varphi(x)}^{\prime \prime}(x) \in C[a, b]$. Then, $u_{\varphi(x)}^{\prime \prime}(x) \in C[a, b]$ and

$$
\begin{aligned}
u_{\varphi(x)}^{\prime \prime}(x)= & K(x, x) u_{\varphi(x)}^{\prime}(x) \\
& +\left[(K(x, x))_{\varphi(x)}^{\prime}+\left.K_{\varphi(x)}^{\prime}(x, s)\right|_{s=x}\right] u(x) \\
& +\int_{a}^{x} K_{\varphi(x)}^{\prime \prime}(x, s) u(s) d \varphi(s)+f_{\varphi(x)}^{\prime \prime}(x),
\end{aligned}
$$

where $x \in[a, b]$. 
In this paper, we assume that $(K(x, x))_{\varphi(x)}^{\prime} \in C[a, b]$, $K_{\varphi(x)}^{\prime \prime}(x, s), K_{\varphi(s)}^{\prime \prime}(x, s) \in C(G)$, and $f_{\varphi(x)}^{\prime \prime}(x) \in C[a, b]$. Then, using Theorems 4 and 5 (and Corollaries 6 and 7), we show that the number $M$ defined as

$$
M=\sup _{(x, s) \in G}\left|[K(x, s) u(s)]_{\varphi(s)}^{\prime \prime}\right|
$$

can be determined in terms of quantities $\|f(x)\|_{C}$, $\left\|f_{\varphi}^{\prime}(x)\right\|_{C},\left\|f_{\varphi}^{\prime \prime}(x)\right\|_{C},\left\|(K(x, x))_{\varphi(x)}^{\prime}\right\|_{C},\left\|K_{\varphi(x)}^{\prime}(x, s)\right\|_{C}$, $\left\|K_{\varphi(s)}^{\prime}(x, s)\right\|_{C},\left\|K_{\varphi(x)}^{\prime \prime}(x, s)\right\|_{C}$, and $\left\|K_{\varphi(s)}^{\prime \prime}(x, s)\right\|_{C}$.

Under these circumstances, using Theorem 2 , the integral

$$
\int_{a}^{x} K(x, s) u(s) d s
$$

can be evaluated numerically by employing the generalized trapezoid rule.

\section{Numerical Solution}

In order to obtain the approximate solution of (1), we employ the generalized trapezoid rule given in [15] to the integral in (1). Let $n \in N$,

$$
\begin{aligned}
h & =\frac{b-a}{n}, \\
x_{k} & =a+k h,
\end{aligned}
$$

where $k=0,1, \ldots, n$. Let us substitute $x=x_{k}$ in the integral equation (1) and examine the following system of equations:

$$
\begin{aligned}
& u\left(x_{0}\right)=f\left(x_{0}\right), \quad x_{0}=a, \\
& u\left(x_{k}\right)=\int_{a}^{x_{k}} K\left(x_{k}, s\right) u(s) d s+f\left(x_{k}\right),
\end{aligned}
$$

$$
k=1,2, \ldots, n .
$$

To evaluate the integral term in (17), we employ the generalized trapezoid rule given in [15] at the nodes $x_{0}, x_{1}, \ldots, x_{k}$. So, we get

$$
\begin{aligned}
\int_{a}^{x_{k}} K\left(x_{k}, s\right) u(s) d s \\
=\sum_{j=1}^{k} \frac{1}{2}\left[K\left(x_{k}, x_{j-1}\right) u\left(x_{j-1}\right)+K\left(x_{k}, x_{j}\right) u\left(x_{j}\right)\right] \\
\quad \cdot\left[\varphi\left(x_{j}\right)-\varphi\left(x_{j-1}\right)\right]+\sum_{j=1}^{k} R_{j}^{(n)}(u),
\end{aligned}
$$

where

$$
\begin{aligned}
\left|R_{j}^{(n)}(u)\right| \leq \frac{M}{12}\left[\varphi\left(x_{j}\right)-\varphi\left(x_{j-1}\right)\right]^{3} & \\
M & =\sup _{(x, s) \in G}\left|[K(x, s) u(s)]_{\varphi(s)}^{\prime \prime}\right| \\
& =\sup _{(x, s) \in G} \mid K(x, s) u_{\varphi(s)}^{\prime \prime}(s)+2 K_{\varphi(s)}^{\prime}(x, s) u_{\varphi(s)}^{\prime}(s) \\
& +K_{\varphi(s)}^{\prime \prime}(x, s) u(s) \mid .
\end{aligned}
$$

Substituting (18) in (17), we get

$$
\begin{aligned}
& u\left(x_{0}\right)=f\left(x_{0}\right), \quad x_{0}=a, \\
& u\left(x_{k}\right) \\
& =\sum_{j=1}^{k} \frac{1}{2}\left[K\left(x_{k}, x_{j-1}\right) u\left(x_{j-1}\right)+K\left(x_{k}, x_{j}\right) u\left(x_{j}\right)\right] \\
& \cdot\left[\varphi\left(x_{j}\right)-\varphi\left(x_{j-1}\right)\right]+\sum_{j=1}^{k} R_{j}^{(n)}(u)+f\left(x_{k}\right),
\end{aligned}
$$

where $k=1,2, \ldots, n$.

Omitting the terms $\sum_{j=1}^{k} R_{j}^{(n)}(u)$ appearing in each equation of system (21) and $u_{k} \approx u\left(x_{k}\right)$, we obtain

$$
\begin{aligned}
u_{0} & =f\left(x_{0}\right), \quad x_{0}=a, \\
u_{k} & =\sum_{j=1}^{k} \frac{1}{2}\left[K\left(x_{k}, x_{j-1}\right) u\left(x_{j-1}\right)+K\left(x_{k}, x_{j}\right) u\left(x_{j}\right)\right] \\
& \cdot\left[\varphi\left(x_{j}\right)-\varphi\left(x_{j-1}\right)\right]+f\left(x_{k}\right),
\end{aligned}
$$

where $k=1,2, \ldots, n$.

Let us assume that

$$
\alpha=\frac{1}{2} \sup _{x \in[a, b]}|K(x, x)| \omega_{\varphi}(h)<1,
$$

where $\omega_{\varphi}(h)$ denotes the modulus of continuity of the function $\varphi$; that is,

$$
\omega_{\varphi}(h)=\sup _{|x-y| \leq h}|\varphi(x)-\varphi(y)| .
$$

Under condition (23), the system of (22) has a unique solution which is given by the formulas

$$
\begin{aligned}
u_{0}= & f(a), \\
u_{1}= & {\left[1-\frac{1}{2} K\left(x_{1}, x_{1}\right)\left(\varphi\left(x_{1}\right)-\varphi\left(x_{0}\right)\right)\right]^{-1} } \\
\cdot & {\left[\frac{1}{2} K\left(x_{1}, x_{0}\right)\left(\varphi\left(x_{1}\right)-\varphi\left(x_{0}\right)\right) u_{0}+f\left(x_{1}\right)\right], } \\
u_{k}= & {\left[1-\frac{1}{2} K\left(x_{k}, x_{k}\right)\left(\varphi\left(x_{k}\right)-\varphi\left(x_{k-1}\right)\right)\right]^{-1} } \\
& \cdot\left[\frac{1}{2} \sum_{j=1}^{k-1} K\left(x_{k}, x_{j}\right)\left(\varphi\left(x_{j+1}\right)-\varphi\left(x_{j-1}\right)\right) u_{j}\right. \\
& \left.+\frac{1}{2} K\left(x_{k}, x_{0}\right)\left(\varphi\left(x_{1}\right)-\varphi\left(x_{0}\right)\right) u_{0}+f\left(x_{k}\right)\right]
\end{aligned}
$$

for $k=2,3, \ldots, n$.

We give a concrete example below.

Example 8. Let us take the integral equation (1) for $a=0$ and $b=2$ with 


$$
\begin{gathered}
\varphi(x)= \begin{cases}\sqrt{x}, & \text { for } 0 \leq x \leq 1, \\
x, & \text { for } 1<x \leq 2\end{cases} \\
K(x, s)=a_{1}(x) b_{1}(s), \quad a_{1}(x)=\left\{\begin{array}{ll}
2 \sqrt{x}+1, & \text { for } 0 \leq x \leq 1, \\
2 x+1, & \text { for } 1<x \leq 2,
\end{array} b_{1}(s)= \begin{cases}s, & \text { for } 0 \leq s \leq 1, \\
s^{2}, & \text { for } 1<s \leq 2,\end{cases} \right.
\end{gathered}
$$

and using

$$
f(x)= \begin{cases}1-\frac{2}{3} x^{2}-\frac{1}{3} x \sqrt{x}, & \text { for } 0 \leq x \leq 1 \\ 1-\frac{x^{3}}{3}(2 x+1), & \text { for } 1<x \leq 2\end{cases}
$$

It is easily seen that $u(x) \equiv 1, x \in[0,2]$, is the unique solution of the integral equation (1) and the conditions $f_{\varphi(x)}^{\prime \prime}(x) \in$ $C[0,2],(K(x, x))_{\varphi(x)}^{\prime} \in C[0,2], K_{\varphi(x)}^{\prime \prime}(x, s)$, and $K_{\varphi(s)}^{\prime \prime}(x, s) \in$ $C(G)$ hold, where $G=\{(x, s): 0 \leq s \leq x \leq 2\}$.

Using the proposed method of this study, we get the following results. Here, 20 nodes are selected; that is, $n=20$. In Table 1, we give the values of the approximate solution obtained by the proposed method of this study and the error in absolute values at the given nodes.

\section{Estimation of the Error}

In this section, we investigate the problem of convergence of the approximate solution $u_{k}$ to the solution of integral (1) at the nodes as $n \rightarrow \infty$.

Theorem 9. Let $\varphi(x)$ be a strictly increasing continuous function on $[a, b]$ and for all $x, y \in[a, b]$ the following inequality holds:

$$
|\varphi(x)-\varphi(y)| \leq L|x-y|
$$

where $L>0$ and $L$ is independent of the variables $x$ and $y$. Then, the inequality

$$
\begin{aligned}
& \left|u\left(x_{k}\right)-u_{k}\right| \\
& \leq \frac{M L^{2}[\varphi(b)-\varphi(a)]}{12(1-\alpha)} \exp \left\{\frac{K_{0} L(b-a)}{1-\alpha}\right\} h^{2}, \\
& k=1,2, \ldots, n,
\end{aligned}
$$

holds in which $K_{0}=\|K(x, s)\|_{C}, \alpha=(1 / 2)\|K(x, x)\|_{C} L h<1$, and the number $M$ is determined by (20).
Proof. Let the error be denoted by $v_{k}=u\left(x_{k}\right)-u_{k}$ for $k=0,1, \ldots, n$. Taking into account (21) and (22), we have the following system of equations:

$$
\begin{aligned}
v_{0}= & 0 \\
v_{k}= & \sum_{j=1}^{k} \frac{1}{2}\left[K\left(x_{k}, x_{j-1}\right) v_{j-1}+K\left(x_{k}, x_{j}\right) v_{j}\right] \\
& \cdot\left[\varphi\left(x_{j}\right)-\varphi\left(x_{j-1}\right)\right]+\sum_{j=1}^{k} R_{j}^{(n)}(u),
\end{aligned}
$$

where $k=1,2, \ldots, n$.

Rearranging the above system of equations, we get

$$
\begin{aligned}
(1- & \left.\frac{1}{2} K\left(x_{1}, x_{1}\right)\left[\varphi\left(x_{1}\right)-\varphi\left(x_{0}\right)\right]\right) v_{1}=R_{1}^{(n)}(u), \\
(1- & \left.\frac{1}{2} K\left(x_{k}, x_{k}\right)\left[\varphi\left(x_{k}\right)-\varphi\left(x_{k-1}\right)\right]\right) v_{k} \\
= & \frac{1}{2} \sum_{j=1}^{k-1} K\left(x_{k}, x_{j}\right)\left[\varphi\left(x_{j+1}\right)-\varphi\left(x_{j-1}\right)\right] v_{j} \\
& +\sum_{j=1}^{k} R_{j}^{(n)}(u)
\end{aligned}
$$

where $k=1,2, \ldots, n$.

Along with the inequality $\omega_{\varphi}(h) \leq L h$, using conditions (19) and (23), we get the following inequality for $v_{k}$ from (31):

$$
\begin{aligned}
& \left|v_{1}\right| \leq \frac{1}{1-\alpha} R(h), \\
& \left|v_{k}\right| \leq \frac{1}{1-\alpha}\left[R(h)+K_{0} L h \sum_{j=1}^{k-1}\left|v_{j}\right|\right],
\end{aligned}
$$

where $k=1,2, \ldots, n, R(h)=(M / 12) L^{2} h^{2}[\varphi(b)-\varphi(a)]$.

Let the term $\varepsilon_{k}$ for $k=1,2, \ldots, n$ be determined by

$$
\varepsilon_{k}=\frac{1}{1-\alpha}\left[R(h)+K_{0} L h \sum_{j=1}^{k-1} \varepsilon_{j}\right], \quad k=2,3, \ldots, n
$$

and $\varepsilon_{1}=R(h) /(1-\alpha)$ as an initial condition.

It is easily seen that $\left|v_{k}\right| \leq \varepsilon_{k}$ for $k=1,2, \ldots, n$. This can be verified by mathematical induction as follows: for $k=1$, it is 
TABLE 1: The values of approximate solution, analytical solution, and the error at the nodes.

\begin{tabular}{|c|c|c|c|}
\hline $\begin{array}{l}\text { The } \\
\text { nodes } \\
x_{k}\end{array}$ & $\begin{array}{l}\text { Real value at } x_{k} \\
\qquad u\left(x_{k}\right)\end{array}$ & $\begin{array}{c}\text { Approx. value at } x_{k} \\
u_{k}\end{array}$ & $\begin{array}{c}\text { The error at } x_{k} \\
\left|u\left(x_{k}\right)-u_{k}\right|\end{array}$ \\
\hline 0 & 1 & 1 & 0 \\
\hline 0.1 & 1 & 1.00271872 & 0.00271872 \\
\hline 0.2 & 1 & 1.00331826 & 0.00331826 \\
\hline 0.3 & 1 & 1.00376994 & 0.00376994 \\
\hline 0.4 & 1 & 1.00417187 & 0.00417187 \\
\hline 0.5 & 1 & 1.00455757 & 0.00455757 \\
\hline 0.6 & 1 & 1.00494416 & 0.00494416 \\
\hline 0.7 & 1 & 1.00534264 & 0.00534264 \\
\hline 0.8 & 1 & 1.00576140 & 0.00576140 \\
\hline 0.9 & 1 & 1.00620761 & 0.00620761 \\
\hline 1.0 & 1 & 1.00668805 & 0.00668805 \\
\hline 1.1 & 1 & 1.00720950 & 0.00720950 \\
\hline 1.2 & 1 & 1.00777907 & 0.00777907 \\
\hline 1.3 & 1 & 1.00840442 & 0.00840442 \\
\hline 1.4 & 1 & 1.00909399 & 0.00909399 \\
\hline 1.5 & 1 & 1.00985722 & 0.00985722 \\
\hline 1.6 & 1 & 1.01070480 & 0.01070480 \\
\hline 1.7 & 1 & 1.01164880 & 0.01164880 \\
\hline 1.8 & 1 & 1.01270300 & 0.01270300 \\
\hline 1.9 & 1 & 1.01388350 & 0.01388350 \\
\hline 2.0 & 1 & 1.01520860 & 0.01520860 \\
\hline
\end{tabular}

trivial. Let $\left|v_{j}\right| \leq \varepsilon_{j}$ for $j=1, \ldots, k-1$. Then, using inequality (32), we get

$$
\left|v_{k}\right| \leq \frac{1}{1-\alpha}\left[R(h)+K_{0} L h \sum_{j=1}^{k-1} \varepsilon_{j}\right]=\varepsilon_{k} .
$$

Let us show that

$$
\varepsilon_{j}=\frac{R(h)}{1-\alpha}\left(1+\frac{K_{0} L h}{1-\alpha}\right)^{j-1}, \quad j=1,2, \ldots, n,
$$

are the solution of the system of (33). Taking (35) into account, we get

$$
\begin{aligned}
& \frac{1}{1-\alpha}\left[R(h)+K_{0} L h \sum_{j=1}^{k-1} \varepsilon_{j}\right] \\
& =\frac{R(h)}{1-\alpha}\left\{1+\frac{K_{0} L h}{1-\alpha} \sum_{j=1}^{k-1}\left(1+\frac{K_{0} L h}{1-\alpha}\right)^{j-1}\right\} \\
& =\frac{R(h)}{1-\alpha}\left\{1+\left[\left(1+\frac{K_{0} L h}{1-\alpha}\right)^{k-1}-1\right]\right\}=\varepsilon_{k},
\end{aligned}
$$

Here, we use the equality

$$
(1+\gamma)^{k-1}-1=\gamma \sum_{j=1}^{k-1}(1+\gamma)^{j-1}, \quad k \geq 2,
$$

where $\gamma=K_{0} L h /(1-\alpha)$. Consequently, we get the following estimate for the error $v_{k}$ for all values $k=1, \ldots, n$ :

$$
\left|v_{k}\right| \leq \frac{R(h)}{1-\alpha}\left(1+\frac{K_{0} L h}{1-\alpha}\right)^{k-1} .
$$

Using the fact that $(1+t)^{1 / t}$ is increasing and approaches the number $e$ as $t \rightarrow 0+$, we get the following chain of inequalities:

$$
\begin{aligned}
(1 & \left.+\frac{K_{0} L h}{1-\alpha}\right)^{k-1} \leq\left(1+\frac{K_{0} L h}{1-\alpha}\right)^{(b-a) / h} \\
& =\left[\left(1+\frac{K_{0} L}{1-\alpha} h\right)^{(1-\alpha) / K_{0} L h}\right]^{K_{0} L(b-a) /(1-\alpha)} \\
& \leq e^{K_{0} L(b-a) /(1-\alpha)}
\end{aligned}
$$

for $k \leq n=(b-a) / h$. Hence, the proof is obtained.

Remark 10. The function

$$
\varphi(x)= \begin{cases}x, & \text { for } 0 \leq x \leq 1 \\ 2 x-1, & \text { for } 1<x \leq 2 \\ 3 x-3, & \text { for } 2 \leq x \leq 3\end{cases}
$$

is a strictly increasing continuous function on $[0,3], \varphi^{\prime}(x) \notin$ $C[0,3]$. But, for all $x, y \in[0,3]$, the following inequality holds:

$$
|\varphi(x)-\varphi(y)| \leq 4|x-y|
$$

Theorem 11. Let $\varphi(x)$ be a strictly increasing continuous function on $[a, b]$ and

$$
\beta=K_{0}[\varphi(b)-\varphi(a)]<1 .
$$

Then, the inequality

$$
\begin{array}{r}
\left|u\left(x_{k}\right)-u_{k}\right| \leq \frac{M}{12(1-\beta)}\left(\omega_{\varphi}(h)\right)^{2}[\varphi(b)-\varphi(a)], \\
k=1,2, \ldots, n,
\end{array}
$$

holds in which $K_{0}=\|K(x, s)\|_{C}$. 
Proof. Let the error be denoted by $v_{k}=u\left(x_{k}\right)-u_{k}$ and set up the system of equations

$$
\begin{aligned}
v_{1}= & \frac{1}{2} K\left(x_{1}, x_{1}\right)\left[\varphi\left(x_{1}\right)-\varphi\left(x_{0}\right)\right] v_{1}+R_{1}^{(n)}(u), \\
v_{k}= & \sum_{j=1}^{k-1} \frac{1}{2} K\left(x_{k}, x_{j}\right)\left[\varphi\left(x_{j+1}\right)-\varphi\left(x_{j-1}\right)\right] v_{j} \\
& +\frac{1}{2} K\left(x_{k}, x_{k}\right)\left[\varphi\left(x_{k}\right)-\varphi\left(x_{k-1}\right)\right] v_{k} \\
& +\sum_{j=1}^{k} R_{j}^{(n)}(u)
\end{aligned}
$$

for $k=2,3, \ldots, n$. From this system of equations, we get

$$
\begin{aligned}
\left|v_{k}\right| & \leq \frac{1}{2} K_{0} \sup _{j}\left|v_{j}\right| \\
& \cdot\left\{\sum_{j=1}^{k-1}\left[\varphi\left(x_{j+1}\right)-\varphi\left(x_{j}\right)+\varphi\left(x_{j}\right)-\varphi\left(x_{j-1}\right)\right]\right. \\
+ & {\left.\left[\varphi\left(x_{k}\right)-\varphi\left(x_{k-1}\right)\right]\right\}+\frac{M}{12}\left(\omega_{\varphi}(h)\right)^{2}[\varphi(b)} \\
& -\varphi(a)]=\frac{1}{2} K_{0} \sup _{j}\left|v_{j}\right|\left[\varphi\left(x_{k}\right)-\varphi\left(x_{1}\right)\right. \\
+ & \left.\varphi\left(x_{k-1}\right)-\varphi\left(x_{0}\right)+\varphi\left(x_{k}\right)-\varphi\left(x_{k-1}\right)\right] \\
+ & \frac{M}{12}\left(\omega_{\varphi}(h)\right)^{2}[\varphi(b)-\varphi(a)] \leq K_{0} \sup _{j}\left|v_{j}\right|[\varphi(b) \\
& -\varphi(a)]+\frac{M}{12}\left(\omega_{\varphi}(h)\right)^{2}[\varphi(b)-\varphi(a)]
\end{aligned}
$$

for $k=2,3, \ldots, n$. Using condition (42), we get inequality (43). Therefore, Theorem 11 is proved.

\section{Competing Interests}

The authors declare that they have no competing interests.

\section{References}

[1] A. S. Apartsyn, Nonclassical Linear Volterra Equations of the First Kind, VSP, TB Utrecht, The Netherlands, 2003.

[2] A. L. Bukhgeim, Volterra Equations and Inverse Problems, VSP, Utrecht, The Netherlands, 1999.

[3] J. Banaś and D. O’Regan, "Volterra-Stieltjes integral operators," Mathematical and Computer Modelling, vol. 41, no. 2-3, pp. 335344, 2005.

[4] J. Banaś, J. R. Rodriguez, and K. Sadarangani, "On a class of Urysohn-Stieltjes quadratic integral equations and their applications," Journal of Computational and Applied Mathematics, vol. 113, no. 1-2, pp. 35-50, 2000.

[5] L. M. Delves and J. Walsh, Numerical Solution of Integral Equations, Oxford University Press, New York, NY, USA, 1974.
[6] M. Federson and R. Bianconi, "Linear Volterrra-Stieltjes integral equations in the sense of the Kurzweil-Henstock integral," Archivum Mathematicum, vol. 37, no. 4, pp. 307-328, 2001.

[7] M. Federson, R. Bianconi, and L. Barbanti, "Linear Volterra integral equations," Acta Mathematicae Applicatae Sinica, vol. 18, no. 4, pp. 553-560, 2002.

[8] A. D. Polyanin and A. V. Manzhirov, Handbook of Integral Equations, Chapman \& Hall/CRC, Boca Raton, Fla, USA, 2nd edition, 2008.

[9] K. Maleknejad and N. Aghazadeh, "Numerical solution of Volterra integral equations of the second kind with convolution kernel by using Taylor-series expansion method," Applied Mathematics and Computation, vol. 161, no. 3, pp. 915-922, 2005.

[10] M. A. Darwish and J. Henderson, "Nondecreasing solutions of a quadratic integral equation of Urysohn-Stieltjes type," Rocky Mountain Journal of Mathematics, vol. 42, no. 2, pp. 545-566, 2012.

[11] S. A. Isaacson and R. M. Kirby, "Numerical solution of linear Volterra integral equations of the second kind with sharp gradients," Journal of Computational and Applied Mathematics, vol. 235, no. 14, pp. 4283-4301, 2011.

[12] T. Diogo, N. J. Ford, P. Lima, and S. Valtchev, "Numerical methods for a Volterra integral equation with non-smooth solutions," Journal of Computational and Applied Mathematics, vol. 189, no. 1-2, pp. 412-423, 2006.

[13] S. Zhang, Y. Lin, and M. Rao, "Numerical solutions for secondkind Volterra integral equations by Galerkin methods," Applications of Mathematics, vol. 45, no. 1, pp. 19-39, 2000.

[14] A. Asanov, "The derivative of a function by means of an increasing function," Manas Journal of Engineering, no. 1, pp. 18-64, 2001 (Russian).

[15] A. Asanov, M. H. Chelik, and A. Chalish, "Approximating the Stieltjes integral by using the generalized trapezoid rule," Le Matematiche, vol. 66, no. 2, pp. 13-21, 2011.

[16] A. Asanov, "Volterra-stielties integral equations of the second kind and the first kind," Manas Journal of Engineering, no. 2, pp. 79-95, 2002 (Russian). 


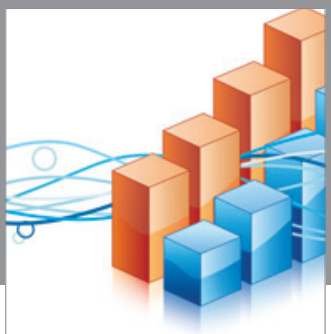

Advances in

Operations Research

vatem alat4

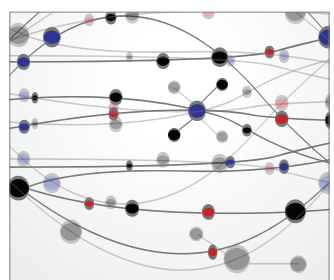

\section{The Scientific} World Journal
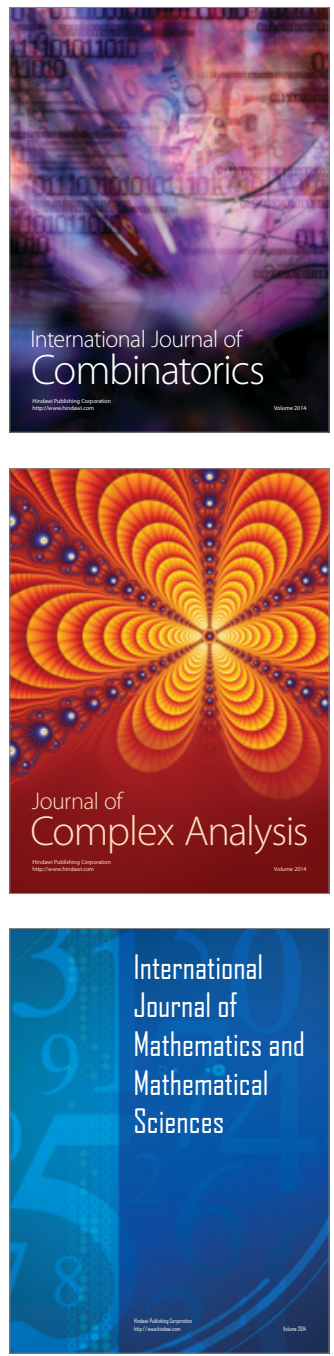
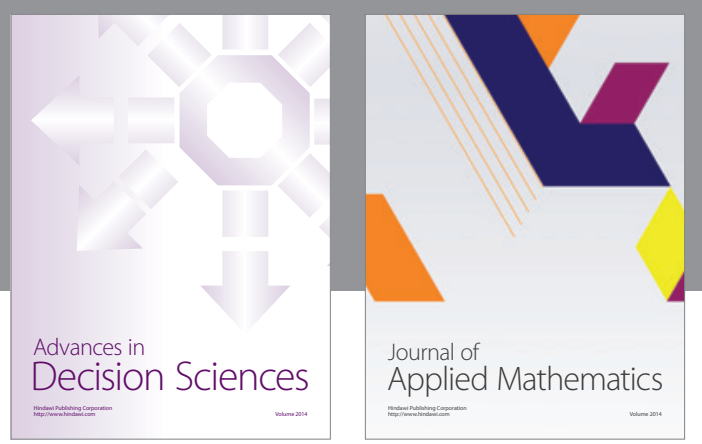

Algebra

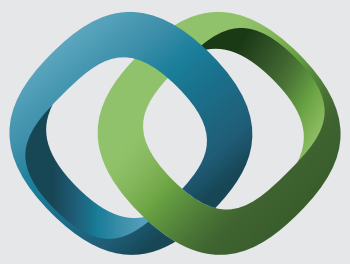

\section{Hindawi}

Submit your manuscripts at

http://www.hindawi.com
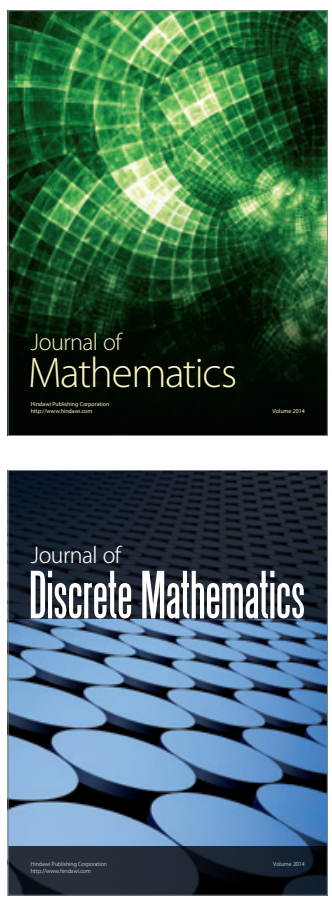

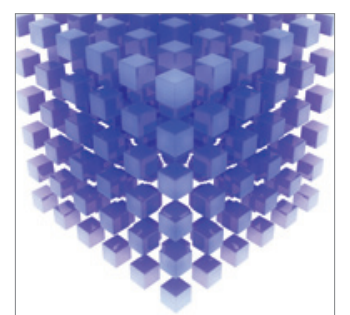

Mathematical Problems in Engineering
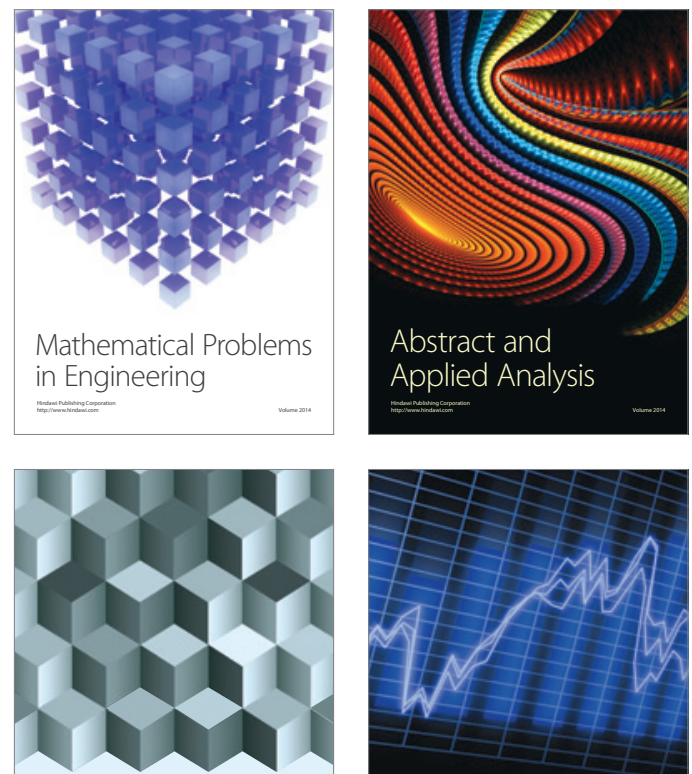

Journal of

Function Spaces

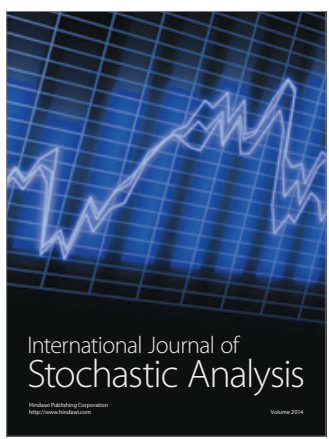

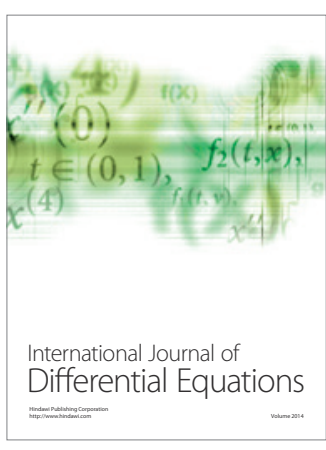
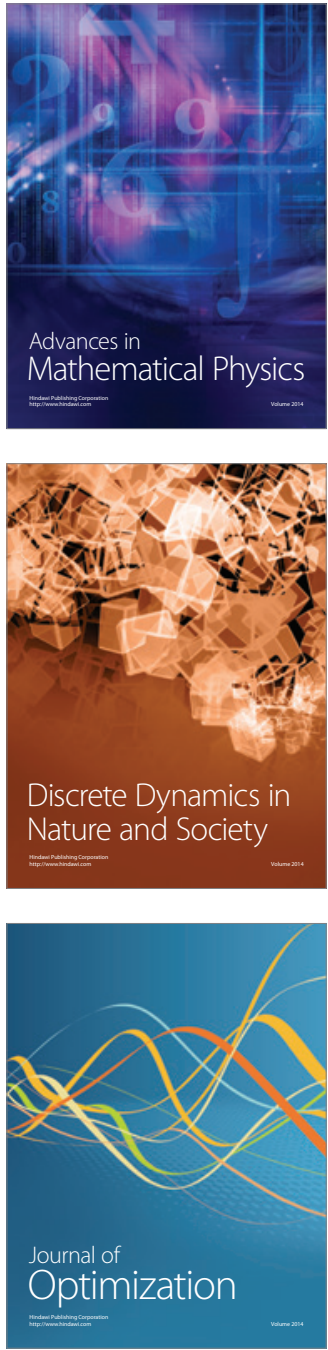